\title{
Influence of air abrasion tips and operation modes on enamel-cutting characteristics
}

Cláudia Peruchi ${ }^{1}$

Ary Santos-Pinto

Tereza Cristina Dias ${ }^{3}$

Ana Carolina Mascarenhas Oliveira ${ }^{4}$

Lourdes Santos-Pinto ${ }^{2}$

\begin{abstract}
Objective: To assess the influence of air abrasion tips and system operation modes on enamel cutting.

Methods: Forty bovine teeth were abraded with the air abrasion system Mach 4.1 for 10 and 15 seconds, employing conventional and sonic tips of $0.45-\mathrm{mm}$ inner diameter and a $90^{\circ}$ angle, and $27.5-\mu \mathrm{m}$ aluminum oxide at $5.51 \mathrm{bar}$ air pressure in continuous and pulsed modes. The width and depth of the resulting cuts were measured in SEM.

Results: The multivariate analysis of variances revealed that, compared to the sonic tip, the conventional tip produced shallower cuts independent of the operation mode and the application period.

Conclusions: The cutting patterns observed in this study suggest that the pulsed mode produced deeper cuts when both the conventional and sonic tips were used, and that the sonic tip cut more dental tissue than the conventional one. (Eur J Dent 2013;7:1-5)
\end{abstract}

Key words: Dental air abrasion; conservative dentistry; dental cavity preparation

\section{INTRODUCTION}

Air abrasion technology is becoming more popular, mainly among pediatric dentists, for cavity preparation. ${ }^{1}$ This technique uses the kinetic energy of aluminum oxide particles that are propelled by compressed air to cut or abrade the target material, and there is no noise to cause discomfort to the professional and to the patient. ${ }^{2-3}$

1 Department of Pediatric Dentistry, Catholic University of Brasilia, BRAZIL

2 Department of Pediatric Dentistry and Orthodontics, Araraquara Dental School, Sao Paulo State University (UNESP), BRAZIL

${ }^{3}$ Department of Statistics, Federal University of Sao Carlos (UFSCar), BRAZIL
The efficiency of tooth tissue removal is relative to the operating parameters of the air abrasion device. Clinicians have to understand the effect of these parameters because they affect the manner in which the tooth tissue is abraded. Alteration of the operative settings can offer a means of further control. The speed of the abrasive particles, de-

- ${ }^{4}$ Department of Health Education, Federal University of Sergipe, BRAZIL

Corresponding author: Dr. Lourdes Santos-Pinto Av. Queiroz Filho, 972 14802-610 - Araraquara - SP, BRAZIL Tel: +55 1633016330 Fax: +5516 33016329

Email: Ispintodfoar.unesp.br 
termined by the air pressure and the distance between the tip and the dental surface, is an important factor in the equipment's cutting efficiency. ${ }^{4}$ The size and type of the abrasive affect the roughness of the abraded surface and, the angle and the inner diameter of the tips determine the profile of the cavity. 5,6 Furthermore, the effects of these parameters are more evident in the cavosurface angles and in the depth of the cavities. Tips with angles close to $80^{\circ}$ produce narrower, more symmetrical, and deeper cuts than do those with angles less than $80^{\circ} .7,8$

The choice of the abrasive unit has a clear effect on the degree of abrasion. ${ }^{6}$ Air abrasion systems available today have either mechanical or digital operator controls. Mechanical control is standard in most devices, and its control of powder flow rate is more tenuous compared to that of digital control, which provides a consistent and minimal amount of powder while maintaining high efficiency. In selected devices, digital control also allows for a pulsed mode of operation, providing an interrupted air abrasive stream at settings from 0.5 to 2.0 seconds.

A majority of the equipment is provided with tips of different angles and inner diameters. However, Mach 4.1 (Kreativ Inc., USA) supplies extra sonic tips that enable doubling the speed of the particles and therefore enhances the speed at which the dental surface is cut.

Since cavity preparation characteristics using the air abrasion system are directly related to the tips and the operation parameters, we aimed to evaluate the effect of 2 air abrasion tips, different operation modes, and varying application times on enamel removal in vitro. The null hypotheses were that there is no detectable cavity preparation difference using conventional or sonic air abrasion tips, and that the operation mode used, as well as the treatment time, does not influence the extent of the cavities prepared with conventional or ultrasonic tips.

\section{MATERIALS AND METHODS}

The dental crowns of 40 bovine incisors were inserted into resin blocks with their vestibular surfaces exposed, and were stored in distilled water with thymol crystals until the beginning of the experiment in order to inhibit possible bacterial activity.

Cuts were made on the enamel surface using the conventional and sonic tips $190^{\circ}$ angle and $0.45-\mathrm{mm}$ inner diameter) coupled with the air abrasion system Mach 4.1 (Kreativ Inc., USA) using aluminum oxide with $27.5-\mu \mathrm{m}$ granulation at 5.51 bar air pressure.

In order to control the variables, the cuts were performed with the help of devices to standardize the position of the specimens and the tip, adapted to a testing machine. These devices enabled placement of the tips $2 \mathrm{~mm}$ from the dental surface and their movement in a straight line at a constant speed $(19.5 \mathrm{~mm} / \mathrm{s})$, as previously described. ${ }^{4}$

Half of each vestibular surface, corresponding to the cervical part of the crown, was covered with wax, and the tip (conventional or sonic) was positioned above the incisal portion. The equipment was then switched on either in the continuous or pulsed mode for 10 seconds, cutting the dental crown in a straight line in the mesiodistal direction. The cut was then covered with wax, and the same tip was positioned on the exposed surface of the tooth with the equipment on for 15 seconds. Therefore, 2 cuts were made on the same specimen with the same tip and operation mode, but for different durations (Table 1).

In an attempt to minimize the effects of the variables inherent to the differences in the den-

Table 1. Experimental conditions per group.

\begin{tabular}{|c|c|c|c|c|}
\hline Equipment & Tip & Operation mode & Application period (s) & Groups \\
\hline \multirow{10}{*}{ Mach 4.1} & \multirow{5}{*}{ Conventional } & \multirow{2}{*}{ Continuous } & 10 & 1 \\
\hline & & & 15 & 2 \\
\hline & & & & 3 \\
\hline & & \multirow{2}{*}{ Pulsed } & 10 & 3 \\
\hline & & & 15 & 4 \\
\hline & \multirow{5}{*}{ Sonic } & \multirow{2}{*}{ Continuous } & 10 & 5 \\
\hline & & & 15 & 6 \\
\hline & & \multirow{3}{*}{ Pulsed } & & 7 \\
\hline & & & 10 & 1 \\
\hline & & & 15 & 8 \\
\hline
\end{tabular}


tal and structural morphologies of the enamel, an equal number of cuts for each tip, mode, and application period were performed in the cervical and incisal parts of the crowns. Each experimental condition was repeated 10 times. In total, 80 cuts were performed.

To assess the cut patterns produced by the different air abrasion tips and parameters, the specimens were sectioned in the cervicoincisal direction with a diamond disk assembled in the cutting machine IsoMet 1000 (Buehler Ltd., Lake Bluff, IL, USA) to obtain 2 fragments from each cut. Each fragment was fixed in isotonic and buffered solutions of aldehyde and osmium tetroxide, dehydrated in increasing concentrations of acetone, critical point-dried to avoid the collapse of the structures present in the specimen surface, and sputtered. The profile and dimension (width and depth) of the cuts were analyzed and measured in SEM.
Multivariate analysis of variance (ANOVA) was employed at a $5 \%$ significance level to compare the effects of the operation mode and the application period of the air abrasion jet to the width and depth of the cuts produced by each tip.

\section{RESULTS}

The average width and depth of the cuts produced using the different settings are presented in Table 2.

The analysis of variance demonstrated no correlation between the operation mode and the application period of the jet to the width and the depth of the cuts when the conventional or sonic tips were employed. However, when the parameters were individually analyzed, the application period of the abrasive jet using the conventional tip influenced neither the width nor depth of the cuts, but the operation mode (continuous or pulsed) in-

Table 2. Width and depth $(\mu \mathrm{m})$ of the cuts under varying operation modes, tips, and application periods.

\begin{tabular}{|c|c|c|c|c|c|c|}
\hline \multirow{2}{*}{ Tip } & \multirow{2}{*}{ Operation mode } & \multirow{2}{*}{ Application period (s) } & \multicolumn{2}{|c|}{ Width $(\mu \mathrm{m})$} & \multicolumn{2}{|c|}{ Depth $(\mu \mathrm{m})$} \\
\hline & & & Average & Standard deviation & Average & Standard deviation \\
\hline \multirow{4}{*}{ Conventional } & \multirow{2}{*}{ Continuous } & 10 & 1135.40 & 284.90 & 50.50 & 36.70 \\
\hline & & 15 & 1098.40 & 217.70 & 58.78 & 20.65 \\
\hline & \multirow{2}{*}{ Pulsed } & 10 & 894.00 & 73.60 & 131.20 & 31.34 \\
\hline & & 15 & 891.60 & 127.60 & 157.20 & 32.70 \\
\hline \multirow{4}{*}{ Sonic } & \multirow{2}{*}{ Continuous } & 10 & 750.10 & 57.20 & 61.00 & 13.66 \\
\hline & & 15 & 741.90 & 47.10 & 83.72 & 14.29 \\
\hline & \multirow{2}{*}{ Pulsed } & 10 & 769.90 & 57.80 & 128.94 & 19.83 \\
\hline & & 15 & 771.70 & 33.30 & 175.10 & 36.30 \\
\hline
\end{tabular}

Table 3. Average values and relative differences in the width and depth $(\mu \mathrm{m})$ of cuts under varying operation modes and application periods with the conventional tip.

\begin{tabular}{|c|c|c|c|c|c|}
\hline \multirow[t]{2}{*}{ Factor } & \multirow[t]{2}{*}{ Variable } & \multicolumn{2}{|c|}{ Average $(\mu \mathrm{m})$} & \multirow[t]{2}{*}{$P$ value } & \multirow[t]{2}{*}{ Relative difference (\%) } \\
\hline & & Pulsed & Continuous & & \\
\hline \multirow[t]{3}{*}{ Mode } & Width & 892.80 & 1116.90 & $.001^{*}$ & 20.06 \\
\hline & Depth & 144.20 & 54.64 & $.000^{*}$ & 62.11 \\
\hline & & $10 \mathrm{sec}$ & $15 \mathrm{sec}$ & & \\
\hline \multirow[t]{2}{*}{ Period } & Width & 1014.70 & 995.00 & .776 & 1.94 \\
\hline & Depth & 90.85 & 107.99 & .072 & 15.87 \\
\hline
\end{tabular}

*Statistical significance $(P \leqslant .05)$

Table 4. Average values and relative differences in the width and depth ( $\mu \mathrm{m})$ of cuts under varying operation modes and application periods using the sonic tip.

\begin{tabular}{|c|c|c|c|c|c|}
\hline \multirow[t]{2}{*}{ Factor } & \multirow[t]{2}{*}{ Variable } & \multicolumn{2}{|c|}{ Average $(\mu \mathrm{m})$} & \multirow[t]{2}{*}{$P$ value } & \multirow[t]{2}{*}{ Relative difference (\%) } \\
\hline & & Pulsed & Continuous & & \\
\hline \multirow[t]{3}{*}{ Mode } & Width & 770.80 & 746.00 & .124 & 3.32 \\
\hline & Depth & 152.02 & 72.36 & $.000^{*}$ & 52.40 \\
\hline & & $10 \mathrm{sec}$ & $15 \mathrm{sec}$ & & \\
\hline \multirow[t]{2}{*}{ Period } & Width & 760.00 & 756.79 & .841 & 0.42 \\
\hline & Depth & 94.97 & 129.40 & $.000^{*}$ & 26.61 \\
\hline
\end{tabular}

*Statistical significance $(P \leqslant .05)$ 
fluenced the depth and the width of the cuts (Table 3). The pulsed operation mode and the 15-second application period influenced the depth of the cuts when the sonic tip was used (Table 4).

Based on the analysis of the average width and depth of the cuts obtained from both tips and the microscopy profile of the cuts, it was noted that the pulsed mode produced deeper cuts with either tip. Additionally, the cuts produced by the sonic tip were deeper than those produced by the conventional tip in both operation modes. We also observed that using the conventional tip resulted in wider cuts as compared to the sonic tips in both operation modes, independent of the application period of the abrasive jet.

\section{DISCUSSION}

The first null hypothesis was rejected, as the results demonstrated significant differences between the cuts produced by the conventional and sonic tips.

The second null hypothesis was also rejected in the case of the conventional tip, as the pulsed operation mode influenced the depth of the cuts. The improved cut efficiency may be attributable to the pulsed mode facilitating the abrasive particles' arrival on the dental surface at a higher speed even at a constant air pressure, possibly due to the interruption of the jet. ${ }^{9}$

Using the conventional tip in continuous mode promoted a slight abrasion on the enamel surface laverage of 1116.90- $\mu \mathrm{m}$ width and $54.64-\mu \mathrm{m}$ depth) (Table 3). Such a setting would be indicated for shallow cuts on the dental surface, such as for the removal of resin or organic plugs in sulci and fissures with a doubtful diagnosis of tooth decay. ${ }^{10,11}$ However, the depth-cutting capacity was increased by $62.11 \%$ (average of $89.56 \mu \mathrm{m}$ ) when the conventional tip was employed in the pulse mode, confirming that there is an increase in the dust flow or the speed of the particles in the pulse mode that enables cutting of the dental tissue in an incremental manner and with more efficiency than that of the continuous mode. ${ }^{9}$

The third hypothesis was not rejected because the application period of the jet did not influence the width and the depth of the cuts, suggesting that at the tip distance used, the maximum efficiency of the cut was achieved following the 10-second application. The width of the cuts is directly related to the distance between the tip of the nozzle and the dental surface. ${ }^{4}$
In case of the sonic tip, the depth of the cut was influenced by the operation mode and the application time. The depth was considerably increased $(52.40 \%)$ when using the pulsed mode, confirming that the pulsed mode is more efficient for cutting dental tissue. It is possible that the intermittent and interrupted aluminum oxide jet $10.5-2.0 \mathrm{sec}-$ onds) increases the speed of the central particles and, consequently, the efficiency of the cut on the dental surface. ${ }^{9,12}$ However, there was only a nonsignificant $(3.22 \%)$ relative difference in the width of the cut (Table 4).

There was a significant increase in the depth of the cuts when the sonic tip was applied for 15 seconds $(P \leqslant .05)$, independent of the operation mode used. This suggests that the particles did not reach their maximum velocity when the sonic tip was applied for 10 seconds, and probably possessed enough energy to go through a longer distance. However, the cutting limit for the width was similar after 10 or 15 seconds, confirming that the central particles of the jet possess a higher cutting capability than those from the periphery. ${ }^{8}$

The sonic tip was more efficient than the conventional tip in terms of the depth of the cut, independent of the mode and the application time (Table 2). This is probably because, in accordance to the manufacturer guidelines, the air in the sonic tips reaches a speed of $400 \mathrm{~m} / \mathrm{s}$ under a 3.45 bar pressure, thus increasing the kinetic energy and, consequently, the cutting performance on the dental surface.

The current study has shown that the operating mode affects the cutting efficiency on enamel. The practical application of these findings is that clinicians can alter the air abrasion equipment parameters to increase cut efficiency, reduce clinical time, and exert greater control over the shape of the cavity created.

\section{CONCLUSION}

The sonic tips cut more dental tissue than the conventional ones did, and the pulsed mode facilitated deeper cuts when using both conventional and sonic tips.

\section{ACKNOWLEDGEMENT}

Special thanks to Professor Doctor José Carlos Fogo, Faculty of Statistics, Federal University of Sao Carlos (UFSCar), for the corroboration in this study. 
Special thanks to Professors Doctors Regina Guenka Palma Dibb, Silmara A. Milori Corona and Maria Cristina Borsatto, Faculty of Dentistry, University of Sao Paulo (USP - Riberao Preto) for lending the Mach 4.1 equipment and for performing the measurements in the optical microscope.

\section{REFERENCES}

1- Leite FRM, Capote TSO, Zuanon, ACC. Application of the total etching technique or self-etching primers. Braz Oral Res 2005;19:198-202.

2- Cook RJ, Azzopardi A, Thompson ID, Watson TF. Real-time confocal imaging during active air abrasion-substrate cutting. J Microsc 2001;203:199-207.

3- Cardenas-Duque LM, Yoshida M, Goto G. Pulpal response to different pulp capping methods after pulp exposure by air abrasion. J Clin Pediatr Dent 2002;26:269-272.

4- Peruchi C, Santos-Pinto L, Santos-Pinto A, Barbosa e Silva E. Evaluation of cutting patterns produced in primary teeth by an air-abrasion system. Quintessence Int 2002;33:279283.

5- Banerjee A, Watson TF, Kidd AM. Dentine caries excavation: a review of current techniques. Br Dent J 2000;188:476-482.

6- Pelka M, Trautmann S, Petschelt A, Lohbauer U. Influence of air-polishing devices and abrasives on root dentin - An in vitro confocal laser scanning microscope study. Quintessence Int 2010;41:e141-e148.

7- Santos-Pinto L, Peruchi C, Marker VA, Cordeiro R. Effect of hand piece tip design on the cutting efficiency of an air abrasion system. Am J Dent 2001;14:397-401.

8- Santos-Pinto L, Peruchi C, Marker VA, Cordeiro R. Evaluation of cutting patterns produced with air-abrasion systems using different tip designs. Oper Dent 2001;26:308312.

9- White JM, Eakle S. Rationale and treatment approach in minimally invasive dentistry. $J$ Am Dent Assoc 2000;131:suppl.:13S-19S.

10- Christensen GJ. Cavity preparation: cutting or abrasion. $J$ Am Dent Assoc 1996;127:1651-1654.

11- Christensen GJ. Air abrasion tooth cutting: state of the art. $J$ Am Dent Assoc 1998;129:484-485.

12- Lambrechts P, Mattar D, De Munck J, Bergmans L, Peumans M, Vanherle G, Van Meerbeek B. Air-abrasion enamel microsurgery to treat enamel white spot lesions of traumatic origin. $J$ Esthet Rest Dent 2002;14:167-187. 